\title{
Molecular Dynamics Simulation of Chain Folding for Polyethylene Subjected to Vibration Excitation
}

\author{
Junfeng Gu, ${ }^{1,2}$ Xue Wang, ${ }^{1}$ Jinying $W u,{ }^{1}$ and Xicheng Wang ${ }^{1}$ \\ ${ }^{1}$ State Key Laboratory of Structural Analysis for Industrial Equipment, Department of Engineering Mechanics, \\ Dalian University of Technology, Dalian 116023, China \\ ${ }^{2}$ Department of Engineering Mechanics, Dalian University of Technology, Dalian 116024, China
}

Correspondence should be addressed to Junfeng Gu; jfgu@dlut.edu.cn

Received 7 March 2014; Accepted 16 July 2014; Published 5 August 2014

Academic Editor: Haojun Liang

Copyright (C) 2014 Junfeng Gu et al. This is an open access article distributed under the Creative Commons Attribution License, which permits unrestricted use, distribution, and reproduction in any medium, provided the original work is properly cited.

\begin{abstract}
We propose a molecular dynamics method with vibration excitation, named as VEMD, to investigate the vibration effect on chain folding for polymer molecule. The VEMD method is based on the introduction of periodic force, the amplitude and frequency of which can be adjusted, and the method was applied to the folding simulation of a polyethylene chain. Simulation results show that the vibration excitation significantly affects the folding of the polyethylene, and frequency and amplitude of the vibration excitation play key roles in VEMD. Different frequencies and amplitudes will determine how and to what extent does the vibration excitation affect the folding process of the polyethylene structure.
\end{abstract}

\section{Introduction}

In Feynman's 1959 lecture named “There's Plenty of Room at the Bottom," he said "ultimately-in the great future-we can arrange the atoms the way we want." However, at the time, the researchers had to accept some atomic arrangement that nature gives [1]. Half a century of progress later, advances in instrumentation have permitted us to observe and characterize materials at atomic scale. New and even more powerful capabilities are rapidly becoming available. Recently, the excitation technique has been applied in polymer processing and has gained more and more interest [2-11]. The types of external excitation include sonic, ultrasonic, electromagnetic, radiation and mechanical vibration depending on their origins. Practice shows that, in the external incentive effect on the polymer density, tensile strength, impact toughness, elastic modulus, thermal properties, brittleness, apparent viscosity, surface roughness, and transparency, even after the crystallization products will impact. By controlling the external excitation can change welding wire, weld line, depression, and stomatal and warp products processing defects and improve the quality of the products. However, most of the achieved results are ambiguous and uncertain due to the fact that the influence mechanism of excitation on the polymer molecule is still not clear. The theoretical understanding and ability to model complex systems have matured to a level that enables the researchers to begin making useful predictions in many areas. Molecular simulation of polymers and polymer analogues has advanced significantly. Refinement of force field parameters, novel simulation methodologies, and the technological revolution in computing power indeed created a paradigm shift. However, to date, some simulations for the mechanical properties of polymer in molding process basically are still limited to the traditional equilibrium molecular dynamics. Research on the influence mechanism needs to investigate the effect of excitation on polymer structure, but simulations under the external excitation on a microscopic level are very rare.

In this work, we propose a molecular dynamics method considering the effect of vibration excitation, named as VEMD, to study the behavior of polymer molecular under vibration. An important aspect of polymer morphology is chain folding, and it is an area of intense activity and debate [12-23]. Therefore, the VEMD method is applied to the folding simulation of a polyethylene chain in this work. The simulation results show that the folding structure of 
polyethylene is significantly affected by the introduced vibration excitation. In addition, the frequency and amplitude of the vibration excitation also have important roles in the folding simulation.

\section{Materials and Methods}

2.1. The VEMD Method. Molecular dynamics (MD) simulation methods solve Newton's equation of motion for a system of $N$ interacting atoms:

$$
m_{i} \frac{\partial^{2} r_{i}}{\partial t^{2}}=F_{i}, \quad i=1, \ldots, N
$$

in which $m_{i}$ and $r_{i}$ are the mass and position vectors of atom $i$, respectively, and $F_{i}$ is the force acting on the atom $i$, which is defined as the negative derivatives of a potential function $V\left(r_{1}, r_{2}, \ldots, r_{N}\right)$ :

$$
F_{i}=-\frac{\partial V}{\partial r_{i}}
$$

Different wave patterns can be chosen for the vibration excitation. The sine wave is a common periodic waveform which occurs often in nature, and it will retain its wave shape when added to another sine wave of the same frequency and arbitrary phase and magnitude. Therefore, a sinusoidal external periodic force is introduced in the molecular dynamics equation:

$$
F_{i}=-\frac{\partial V}{\partial r_{i}}+f_{0} \sin 2 \pi \omega t \cdot \vec{R}
$$

in which $f_{0}$ is the amplitude, $\omega$ is the vibration frequency, and $\vec{R}$ is the direction of the vibration.

\subsection{The Polyethylene Model. To test the effect of external exci-} tation on the polyethylene folding, one linear polyethylene chain containing 1000 backbone carbon atoms is used in this work. The all atom OPLS-AA force field is adopted to simulate the folding process of polyethylene, and default parameters are used $[24,25]$.

Initial structure of the polyethylene chain was in a straight line, and it was energy minimized using the conjugate gradient method. The resultant structure was then subjected to a subsequent $\mathrm{MD}$ simulation in vacuum for as long as $1 \mathrm{~ns}$. The simulation was performed with the MD program GROMACS4.0.5 [26], and the integration time step was $2 \mathrm{fs}$. The system was coupled to a Berendsen thermostat at $463 \mathrm{~K}$ (a temperature commonly used in the characterization of polyethylene liquids in practice), and the coupling time is $0.1 \mathrm{ps}$. There were no periodic boundary conditions and no pressure coupling when simulation is taken in vacuum. The cutoffs for van der Waals and Coulomb interactions were turned off so all interactions were computed. The root mean square deviation (RMSD) curve and the surface area curve of the $1 \mathrm{~ns}$ MD simulation are shown in Figure 1, which indicates that the structure of the polyethylene chain is in a relatively stable state after the first 200 ps simulation. In this work, the
RMSD values were calculated with initial structure as the reference structure, and the surface area values were obtained with a probe ball the radius of which is $0.14 \mathrm{~nm}$.

The final structure of the polyethylene chain after the 1 ns simulation is shown in Figure 2(a), and it was treated as the starting structure of the subsequent conventional molecular dynamics (CMD) and VEMD simulations. All the simulations were performed for $2 \mathrm{~ns}$ with coupling to a Berendsen thermostat at $463 \mathrm{~K}$, and the integration time step was set as $2 \mathrm{fs}$.

\section{Results and Discussion}

3.1. The Influence of the Vibration Frequency on the Polyethylene Folding. For investigating the influence of the vibration frequency, we firstly performed six VEMD simulations with different vibration periods: $0.2 \mathrm{ps}, 0.4 \mathrm{ps}, 0.8 \mathrm{ps}, 2 \mathrm{ps}, 4 \mathrm{ps}$, and $8 \mathrm{ps}$, and the amplitude of all the VEMD simulations was set as $500 \mathrm{~kJ} \mathrm{~mol}^{-1} \mathrm{~nm}^{-1}$. The orientation of the starting structure was along the $z$-direction as shown in Figure 2(a), and the vibration direction was set in the $x$-direction to investigate the influence of the external excitation on the molecular orientation.

The relationship between the vibration frequency and the global structure change was firstly investigated. Surface area of a molecule is an important indicator of the level of chain folding, and its reduction means a more compact and stable folded structure. Figure 3 gives the change curves of surface area during the CMD and VEMD simulations. The CMD simulation is shown in black, and six VEMD simulations are shown in different colors. The black curve keeps a relatively stable trend, which indicates that the 2 ns CMD simulation will not obviously change the folding level of the polyethylene chain. However, as shown in Figure 3, the introduction of external excitation significantly decreases the surface area of the polyethylene structure. When the vibration period is 0.2 ps (the red curve in Figure 3(a)), the surface area gradually decreases during the first 500 ps and keeps at about $90 \mathrm{~nm}^{2}$ afterwards. When the vibration period increases to $0.4 \mathrm{ps}$ (the blue curve in Figure 3(a)), the surface area sharply decreases to $80 \mathrm{~nm}^{2}$ during the first dozens of ps. When the vibration period keeps increasing ( $0.8 \mathrm{ps}, 2 \mathrm{ps}, 4 \mathrm{ps}$, and $8 \mathrm{ps})$, the descending trends of the surface area curves are very similar to the curve of $0.4 \mathrm{ps}$ VEMD simulation, and the surface areas all keep at about $80 \mathrm{~nm}^{2}$. This indicates that when the vibration period exceeds $0.4 \mathrm{ps}$, the external excitation will not further change the folding level.

The final structure of the polyethylene chain after $2 \mathrm{~ns}$ CMD simulation is shown in Figure 2(b). Compared with the starting structure shown in Figure 2(a), the appearance of the molecule has gone through considerable changes, and the structure is not regular with plenty of random coils. The molecular orientation of the final structure of the CMD simulation is still along the $z$-direction, which shows that CMD simulation does not affect the molecular orientation of the polyethylene structure.

The final structures derived from the VEMD simulations are shown in Figure 4 separately, with the vibration period 


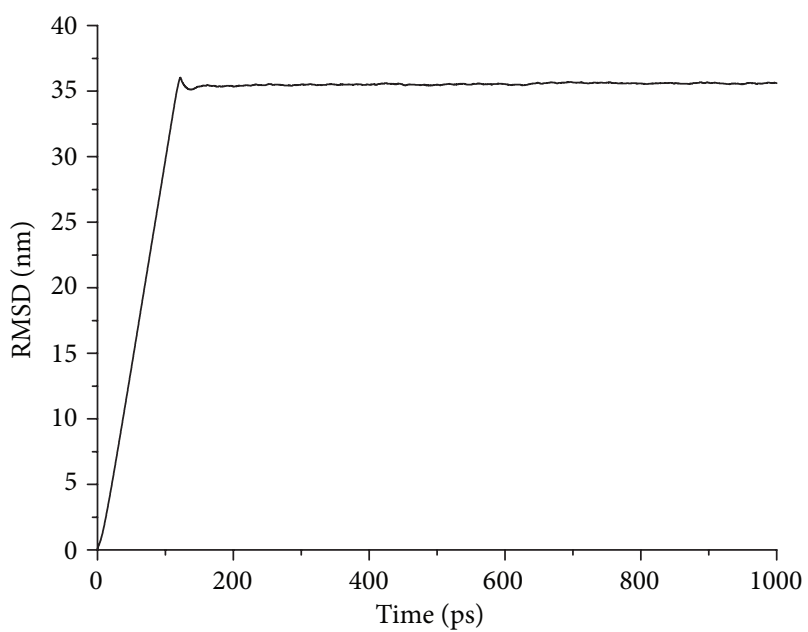

(a)

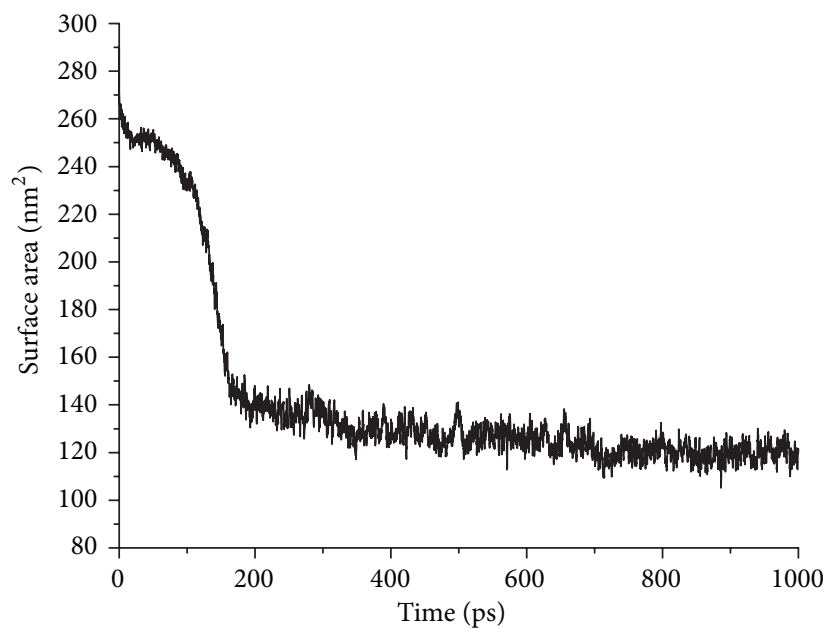

(b)

FIgURE 1: (a) The RMSD curve and (b) the surface area curve of the polyethylene chain during the $1 \mathrm{~ns}$ CMD relaxation simulation.

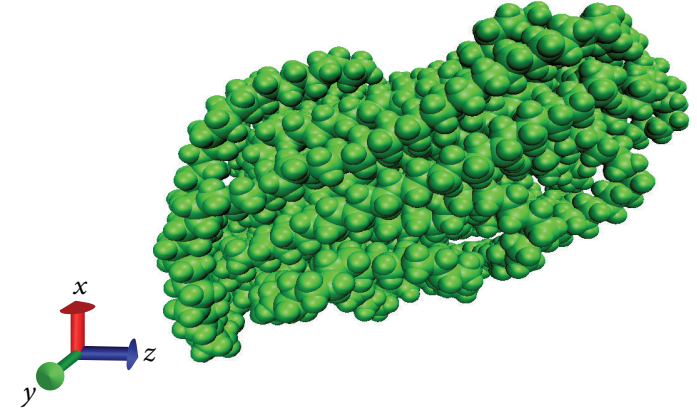

(a)

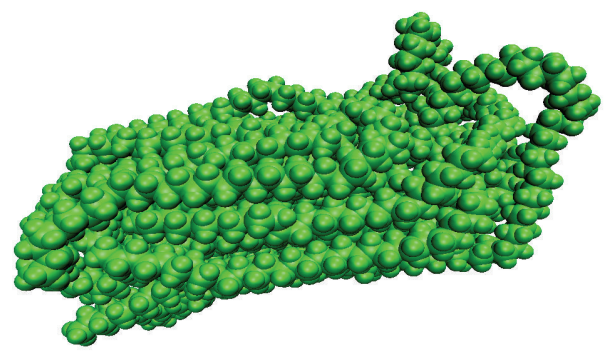

(b)

FIGURE 2: The final structures of the polyethylene chain (a) of the $1 \mathrm{~ns}$ relaxation simulation and (b) of the subsequent 2 ns CMD simulation.

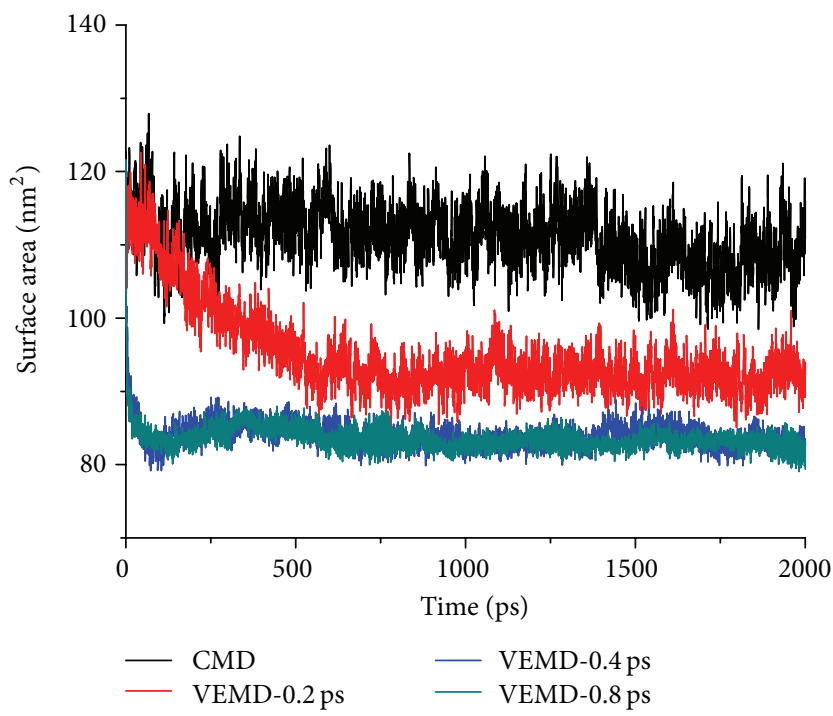

(a)

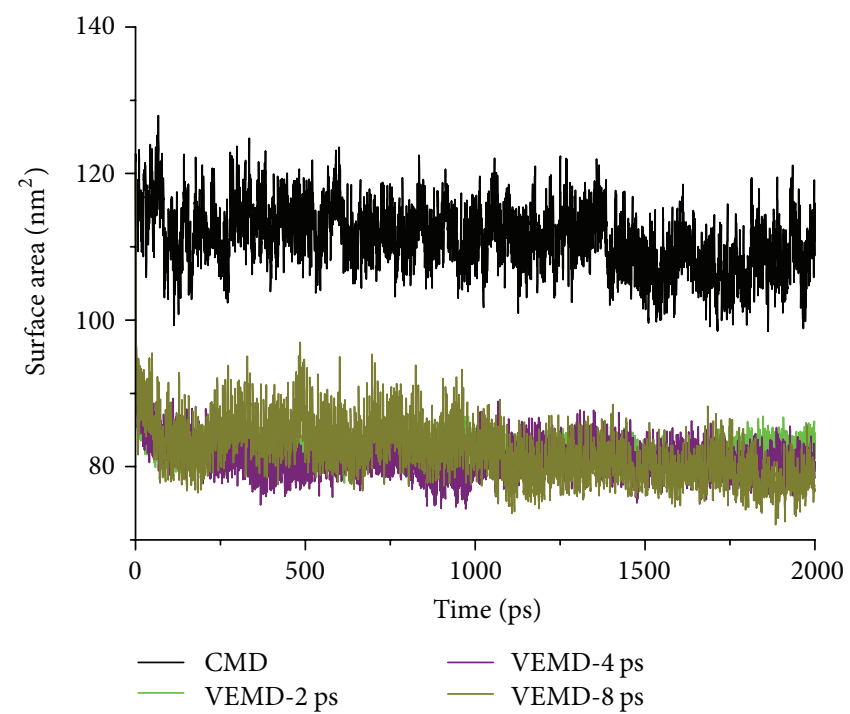

(b)

FIGURE 3: The surface area curves of the polyethylene molecule during the CMD simulation (shown in black) and six VEMD simulations with different vibration periods (shown in colors). 


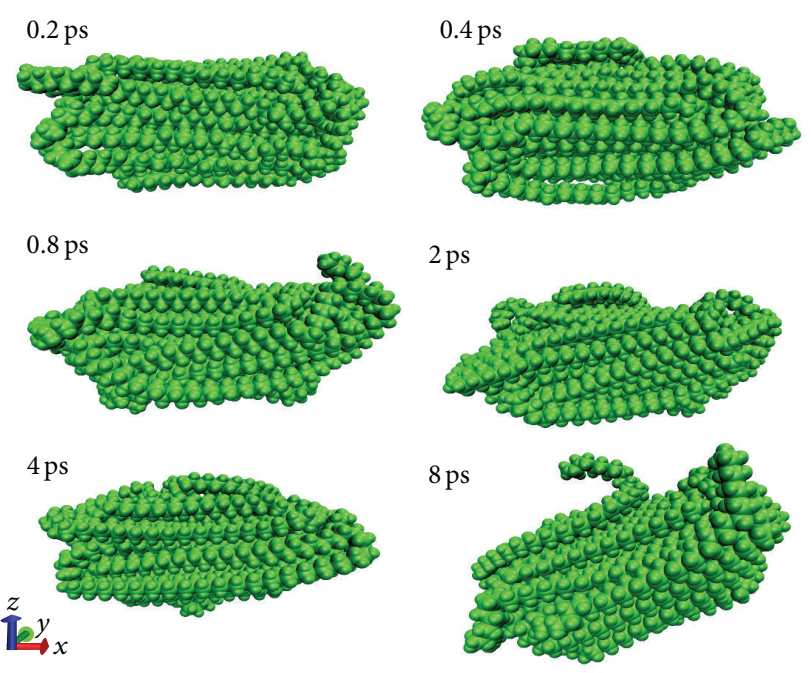

FIGURE 4: The final structures of the VEMD simulations with different vibration periods.
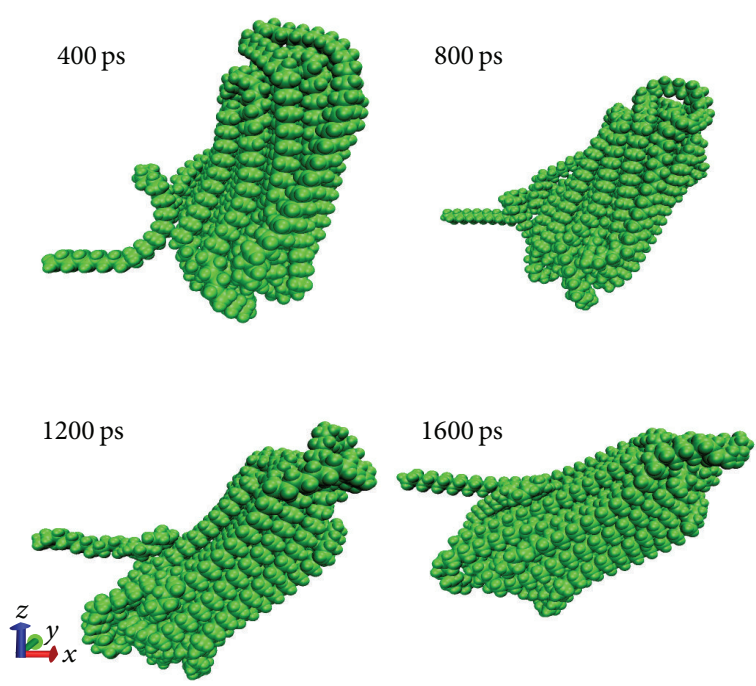

Figure 5: Snapshots at 400 ps, 800 ps, 1200 ps, and 1600 ps during the VEMD simulation with 8 ps vibration period.

labeled at the top left. The orientations of the VEMD final structures are all along the $x$-direction, which means that the vibration direction will affect the molecular orientation in the simulation process. It can be seen that, compared with $\mathrm{CMD}$, the linear chain folded more tightly and regularly with the chain segments aligning as a lamella, and with vibration period increases, the local structures are organized in a more orderly manner. For investigating the influence process of the vibration, Figure 5 gives the snapshots at $400 \mathrm{ps}, 800 \mathrm{ps}, 1200 \mathrm{ps}$, and 1600 ps during the VEMD simulation with 8 ps vibration period. An obvious orientation change of the structure along the vibration direction can be observed clearly from this figure. Meanwhile, the molecule conformation is kept relatively stable during the simulation. From Figures 3-5, it is clear that the external excitation has great impact on the finally folded structure of polyethylene, and the period of the vibration excitation plays an important role in the process.

For further evaluating the structure change caused by vibration excitation, RMSD curves of all the carbon atoms derived from the CMD and VEMD simulations are plotted and compared in Figure 6. As shown in Figure 6, CMD simulation has the largest RMSD value (about $1.75 \AA$ ) after $2 \mathrm{~ns}$ simulation, and the fluctuation of the RMSD curve is also the largest, which indicates that the structure of the polyethylene chain under the CMD simulation is quite flexible and unstable. Figure 6(a) also gives the RMSD curves derived from three VEMD simulations, and the vibration periods are $0.2 \mathrm{ps}, 0.4 \mathrm{ps}$, and $0.8 \mathrm{ps}$, respectively. It can be seen that compared with CMD, the structure of the polyethylene can be stabilized with certain extent when external excitation is introduced. In addition, when the vibration period increases, the RMSD value of the final structure after 2 ns simulation is decreased, and the fluctuation of the RMSD curve is less obvious. This characteristic indicates that the global structure of the polyethylene is better conserved and stabilized when the period of the external excitation increases from $0.2 \mathrm{ps}$ to $0.8 \mathrm{ps}$. However, when the period varies from $2 \mathrm{ps}$ to 8 ps, as shown in Figure 6(b), things will develop in the opposite direction: when the vibration period increases, the RMSD value of the final structure after $2 \mathrm{~ns}$ simulation is increased, and the fluctuation of the RMSD curve becomes more obvious. The reason will be further discussed in the following section.

For analyzing the influence of the external excitation on the local structure of the folded polyethylene, the carbon atom torsion angle distributions of the finally folded structure derived from the CMD and VEMD simulations are statistically analyzed and plotted in Figure 7. Figure 7(a) gives the comparison of CMD and three VEMD simulations (vibration periods are $0.2 \mathrm{ps}, 0.4 \mathrm{ps}$, and $0.8 \mathrm{ps}$, resp.), and Figure $7(\mathrm{~b})$ gives the comparison of CMD and other three VEMD simulations (vibration periods are $2 \mathrm{ps}, 4 \mathrm{ps}$, and 8 ps, resp.). Three peaks are obviously observed in all the distribution curves in Figure 7, which correspond to the three equilibrium states of the dihedral angle: gauche ${ }^{+}\left(\varphi=60^{\circ}\right)$, trans $\left(\varphi=180^{\circ}\right)$, and gauche ${ }^{-}\left(\varphi=300^{\circ}\right)$. It is seen that, in the distribution of the CMD simulation, the distributions of the three equilibrium states are broad: the gauche ${ }^{+}$ranges from 50 to $100^{\circ}$, the trans ranges from 130 to $220^{\circ}$, and the gauche ${ }^{-}$ranges from 250 to $320^{\circ}$, which suggests that the local structures of the polyethylene chain are not so ordered under the CMD simulation. In addition, it is obvious that when the external excitation is introduced, the torsion distribution is affected. When the vibration period of the external excitation is relatively small, the influence is almost negligible. The torsion distribution curve of VEMD is almost identical to the one of the CMD simulation when the vibration period is $0.2 \mathrm{ps}$. However, when the vibration period is $8 \mathrm{ps}$, the distribution ranges of equilibrium states are significantly suppressed, and the trans conformation especially becomes more distinct and narrower than in the case of CMD and other VEMD simulations. As a result, the ratio between the trans/gauche states increases. Analysis of the torsion distribution indicates that relatively high frequency external 


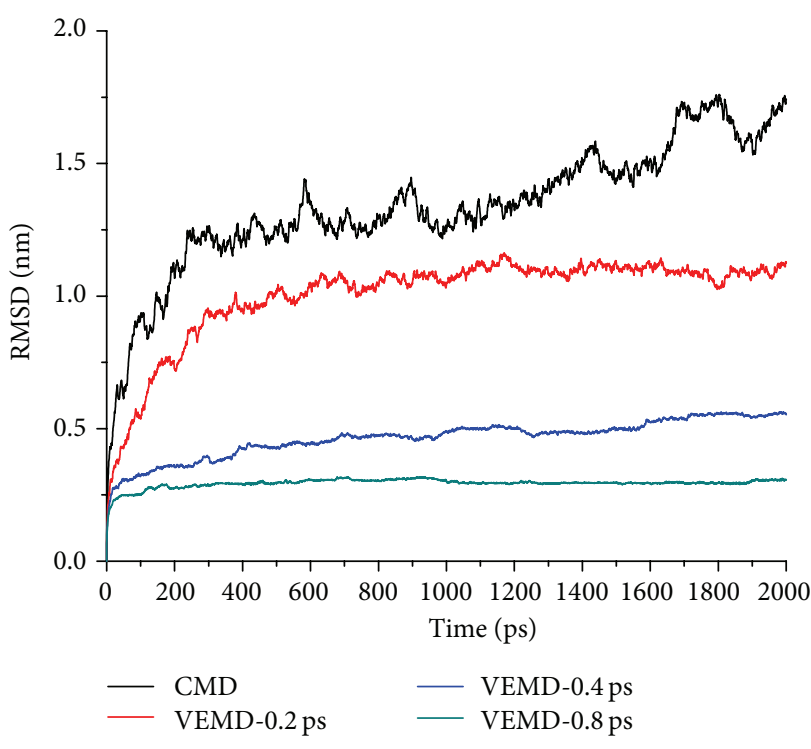

(a)



(b)

Figure 6: The RMSD curves of the CMD and VEMD simulations.

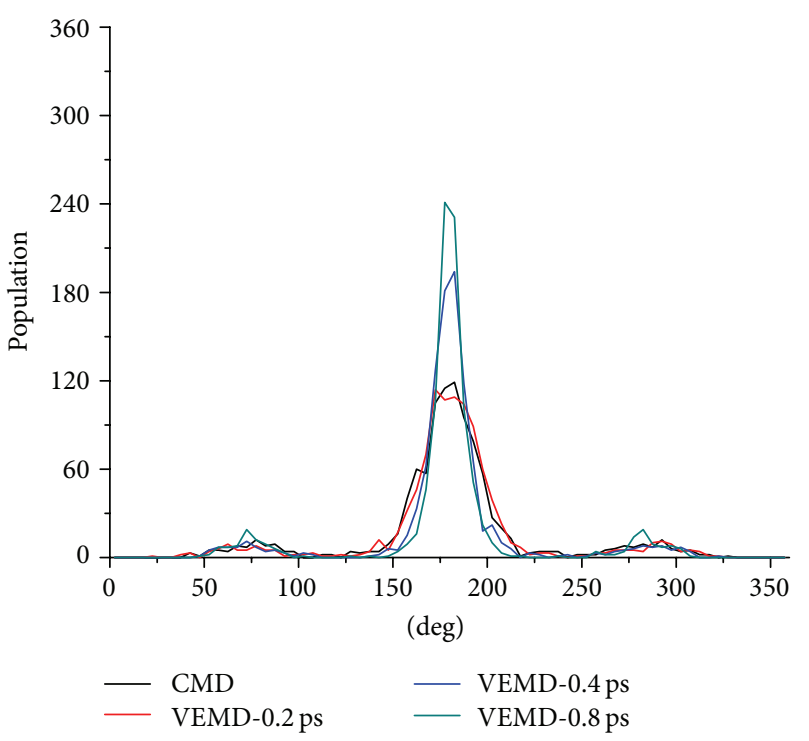

(a)

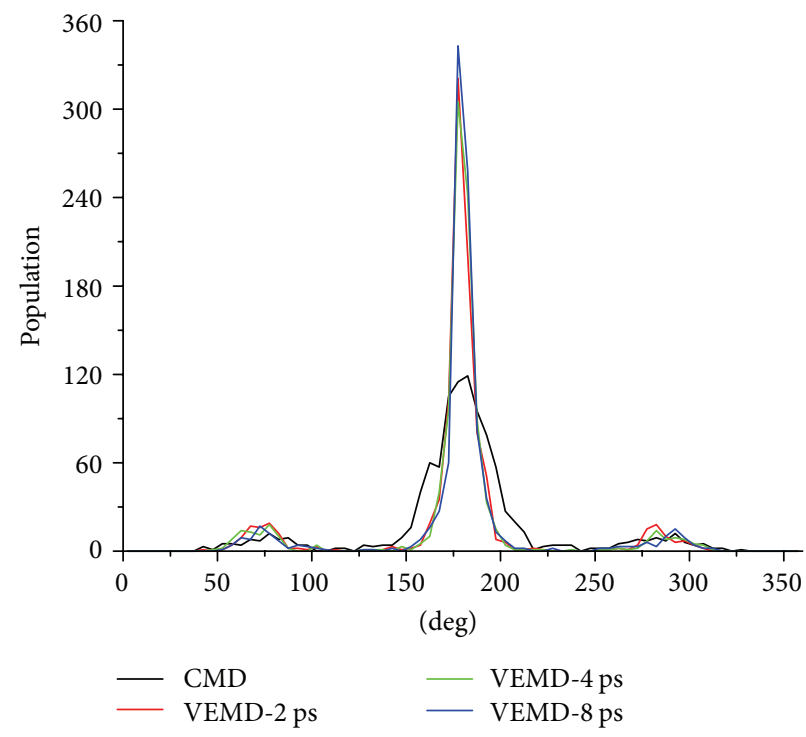

(b)

FIGURE 7: The torsion distributions of the final structures derived from the CMD and VEMD simulations.

excitation will less affect the local structures of polyethylene chain. Along with the growth of the vibration period, the influence becomes increasingly significant.

Combining the analysis of Figures 4-7 and the observation of the simulation trajectories, it is found that when the vibration period is short (e.g., $0.2 \mathrm{ps}$ ), the segments of the polyethylene structure will be stretched along the vibration direction, so the RMSD curve will be relatively high referenced to the starting structure. While with the vibration period increases, the stretching effect of the external excitation will be decreased, and the constraint effect on the global structure will be more obvious, so the RMSD curve will be lowered and stabilized during the simulation (e.g., 0.8 and 2 ps). However, when the vibration period keeps increasing, the RMSD curve is raised again. For analyzing the reason, we further compared the VEMD simulations with 4 and 8 ps vibration periods. In Figure 7, these two simulations have almost identical torsion distributions, but they have obviously different RMSD curves in Figure 6. Therefore, we firstly compared the root mean square fluctuation (RMSF) of all the carbon atoms during these two simulations, as plotted in Figure 8(a). RMSF in the red curve (VEMD-8 ps) has more overall improvement than the black curve (VEMD-4 ps), especially in the segment labeled with dashed blue cycle. 


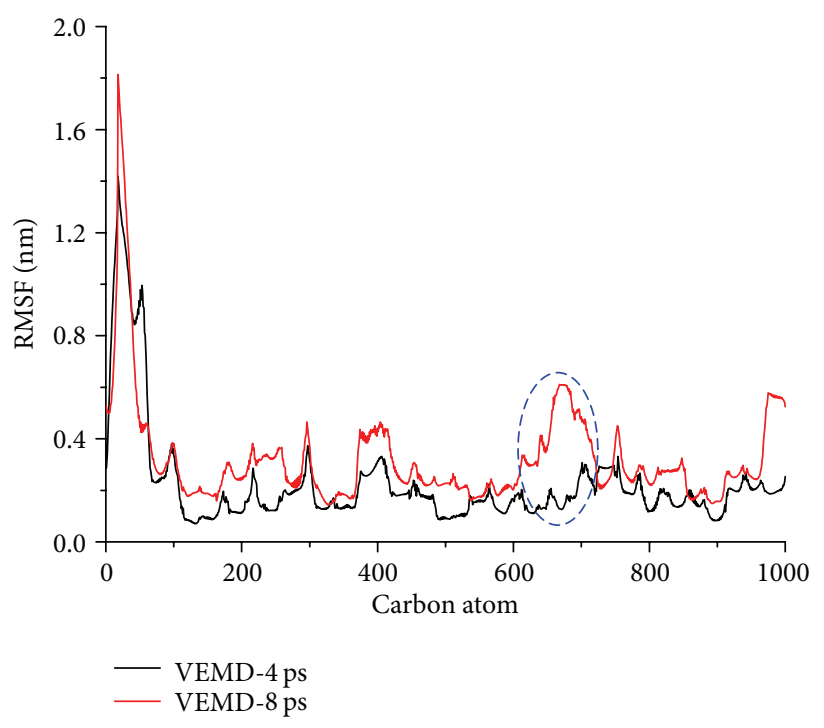

(a)

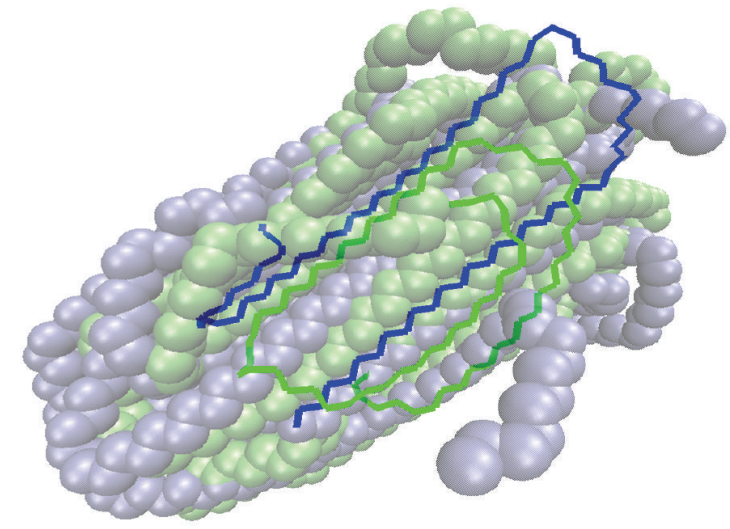

(b)

FIgURE 8: The RMSF values of carbon atoms of the polyethylene molecule (a) and the overlaid final structures (b) of the VEMD simulations with 4 (shown in green in (b)) and 8 ps (shown in blue in (b)) vibration periods.

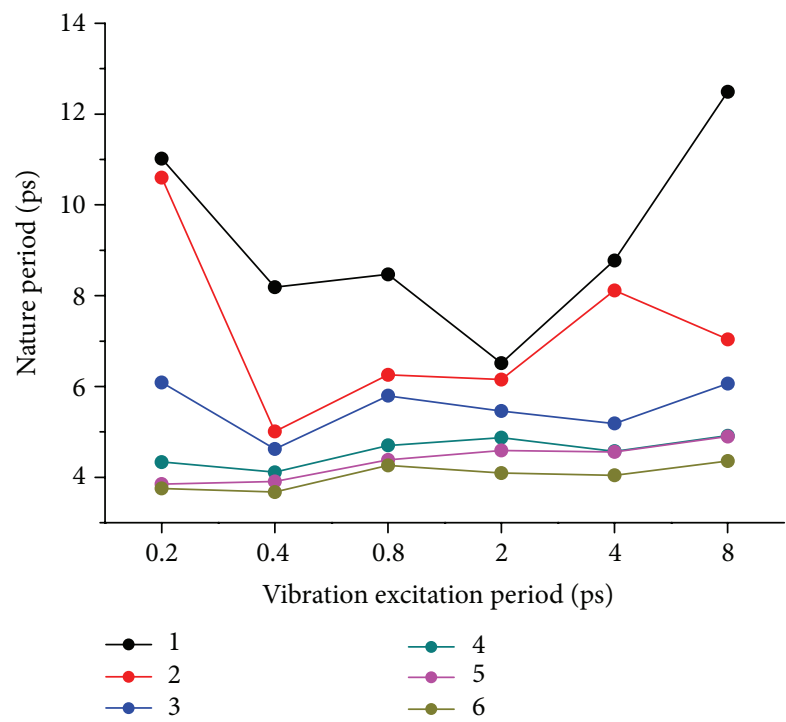

FIGURE 9: The relationship between the vibration periods and the first six order nature periods of the VEMD final structures.

Then, the final structures derived from these two simulations are overlaid and shown in Figure 8(b). The structure derived from VEMD-4 ps is shown in green, and the structure of VEMD-8 ps is shown in blue. For directly investigating the segments labeled with the dashed cycle in Figure 8(a), they are drawn with lines in Figure 8(b). It is seen that the RMSF values of the segment in the black curve of Figure 8(a) are relatively low, which indicates that it is less affected by the vibration with 4 ps period, and the segment structure of VEMD-4 ps (shown in green line in Figure $8(\mathrm{~b})$ ) is on the surface of the polyethylene structure, and it is composed of abnormal short lamella. However, when the vibration period increases to $8 \mathrm{ps}$, the segment is significantly affected by the external excitation, and the final segment structure is folded to more regular long lamella. Differences in this kind of segments will cause the difference between the RMSD curves of VEMD- 4 ps and VEMD-8 ps. From the abovementioned results, we can conclude that when the vibration period increases to a certain extent, the constraint on the global structure of the polyethylene will be loose and local segments will be affected and reorganized to a more regular structure, and this will eventually increase the RMSD value of the simulation.

Natural modes indicate the structural and vibrational characteristics of molecular structure. For deeply discussing the effect of the external excitation on the polymer folding, we also analyzed the influence of the frequency of external vibration on the modes of the molecule. Normal mode analysis was firstly taken on the starting structure. As the first few principal modes often describe collective, global motions in the system, only the first six order periods are analyzed here, and the values of them for the starting structure are $9.04,7.42,6.14,5.64,5.37$, and $5.11 \mathrm{ps}$, respectively. The modes of the final structures of the VEMD simulations were also calculated. Figure 9 gives the first six order nature periods of the VEMD final structures obtained under different vibration frequencies. As can be seen from Figure 9 the frequency significantly influences the normal modes of the final structure of the VEMD, especially for the first two order nature periods. For the first order nature period, the value is lowest when the vibration period is $2 \mathrm{ps}$. When compared with the starting structure, the first order nature period is decreased when the vibration period is $0.4,0.8$, and 2 ps. While for the higher order nature periods, the 0.4 ps vibration period has the most obvious influence. All the values 


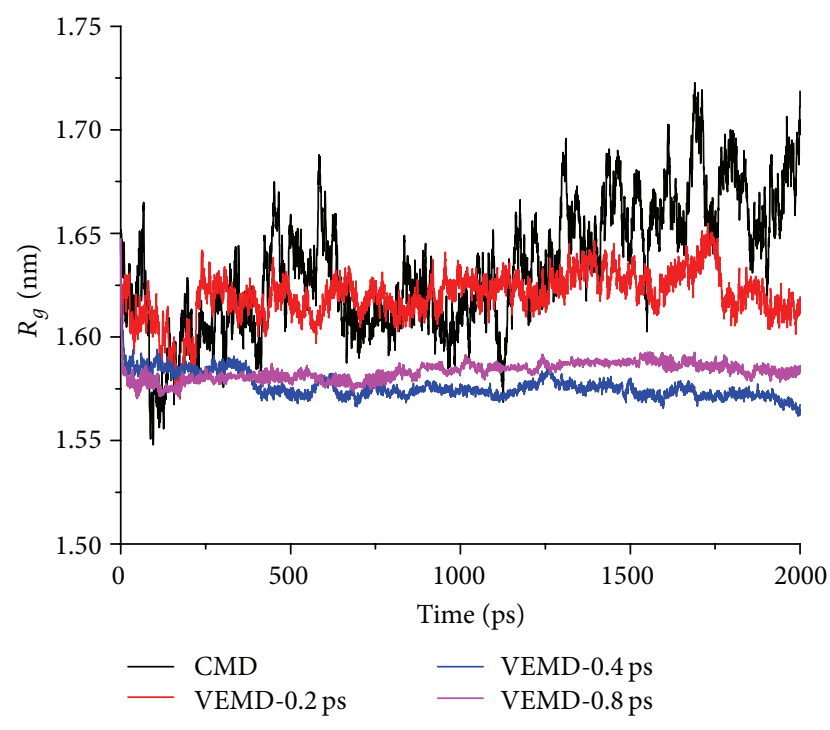

(a)

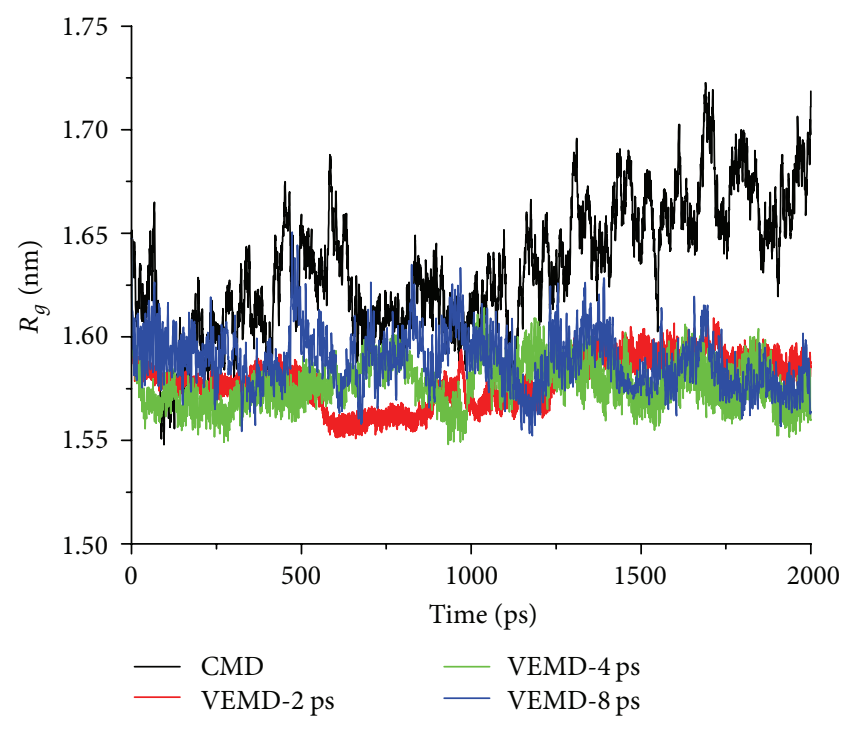

(b)

FIgURE 10: Radius of gyration curves of the CMD and VEMD simulations.

of the 4-6 order periods of the VEMD structures are decreased when compared with the starting structure. The results show that the vibration period can influence the nature vibration modes of the final structure, and the reason is that vibration excitation can affect both the global and local structures of the polymer molecule as the abovementioned analysis reveals. In other words, with different vibration periods we can get folded polymer structures with different nature vibration characteristics.

To have a good understanding of how much the individual molecule change during the simulation process, radius of gyration Rg curves was calculated with the tool provided in GROMACS4.0.5 package and given in Figure 10. Rg curve of the CMD simulation is shown in black line, and the values are $1.64 \pm 0.1 \mathrm{~nm}$, and there is a slight rising after $1000 \mathrm{ps}$. $\mathrm{Rg}$ curve of EEMD-0.2 ps is similar to the CMD, except it is more stable, and the $\mathrm{Rg}$ values are $1.62 \pm 0.05 \mathrm{~nm}$. Rg curves of EEMD-0.4 ps and EEMD-0.8 ps are similar. They have a rapid decrease from $1.65 \mathrm{~nm}$ to about $1.58 \mathrm{~nm}$ at the beginning of the simulations and keep stable afterwards. $\mathrm{Rg}$ curves of EEMD-2 ps, EEMD-4 psm, and EEMD-8 ps also have large decreases during the simulations, but the fluctuations of them are comparatively large when referenced to the $\mathrm{Rg}$ curve of EEMD-0.4 ps and EEMD-0.8 ps. The results show that vibration excitation has an obvious influence on the radius of gyration of the simulated structure. With the introduction of vibration, the compactness of the structure is improved, and the influence extent varies with different vibration frequencies.

\subsection{The Influence of the Vibration Amplitude on the Polyethy-} lene Folding. Amplitude is a measure of the severity of the vibration. For evaluating the influence of the vibration magnitude to the polymer folding, we took another simulation experiment by fixing the vibration period but varying the amplitude meanwhile. In the previous chapter about vibration frequency, the 2 ps period has a significant influence on the polymer, so 2 ps is set as the fixed vibration period in this chapter. Except for the abovementioned simulation with $500 \mathrm{~kJ} \mathrm{~mol}^{-1} \mathrm{~nm}^{-1}$ amplitude, another five simulations were performed, and their amplitudes were 250, 750, 1000, 1250, and $1500 \mathrm{~kJ} \mathrm{~mol}^{-1} \mathrm{~nm}^{-1}$, respectively.

Similarly, the RMSD curves of these simulations were calculated and compared, as plotted in Figure 11. When the amplitude is small $\left(250 \mathrm{~kJ} \mathrm{~mol}^{-1} \mathrm{~nm}^{-1}\right)$, the mean value and the fluctuation of the RMSD curve of the simulation are obviously the biggest; however, when the amplitude was changed to 500 and $750 \mathrm{~kJ} \mathrm{~mol}^{-1} \mathrm{~nm}^{-1}$, the RMSD curves were lowered and stabilized. When the amplitude keeps increasing $\left(1000,1250\right.$, and $\left.1500 \mathrm{~kJ} \mathrm{~mol}^{-1} \mathrm{~nm}^{-1}\right)$, the RMSD curves begin to rise again. Figure 11 indicates that when the vibration magnitude is relatively small, the constraint caused by the vibration on the polyethylene structure is also small, and the RMSD curve is relatively high. When the amplitude reaches a certain value, the constraint will keep increasing, and the RMSD curve reaches the lowest position. However, if the amplitude exceeds the certain value and the vibration becomes more intense, it will result in the structural change and the rise of the RMSD curve.

The torsion distributions of these simulations were also analyzed, as plotted in Figure 12. Compared with the torsion distribution of CMD in Figure 7, VEMD simulations with varied amplitudes also improve the torsion distribution. In addition, different amplitude will cause differences in torsion distribution. When the amplitude is $500 \mathrm{~kJ} \mathrm{~mol}^{-1} \mathrm{~nm}^{-1}$, the torsion distribution is relatively regular as indicated in Figure 7. However, when the amplitude is $250 \mathrm{~kJ} \mathrm{~mol}^{-1} \mathrm{~nm}^{-1}$, although the RMSD values were relatively high as shown in Figure 11, the regularity of the torsion distribution is further improved. Other amplitudes slightly reduce the regularity, 
but the differences are not as obvious as the ones in Figure 7 which are caused by varied vibration frequencies. Analysis of the torsion distribution indicates that appropriate amplitude will further improve the regularity of the local structures of polyethylene chain.

From Figures 11-12, vibration amplitude also plays an important role in the polymer folding, although the role is not as significant as the vibration frequency. With varying amplitude, the response of the polymer structure is different. However, through the analysis of the figures, linear relations between the amplitude and the polymer response are not observed. How to choose appropriate combination of vibration frequency and amplitude to get a wanted response of polymer structure is a subject that needs to be further researched.

\section{Conclusions}

For evaluating the effect of vibration excitation on the folding of polymer molecule, we introduced a vibration excitation molecular dynamics simulation method, named as VEMD, and this method was applied to the folding simulation of polyethylene molecule that consisted of 1000 carbon atoms. The results show that the vibration excitation has an obvious influence on the polymer chain folding. Frequency of the vibration excitation has a key role in the simulation. When the frequency is relatively high, the vibration excitation will have stretching effect on the polyethylene structure, and with the decrease of the frequency, constraint effect will be increasingly significant. By then, the global structure of the polyethylene is constrained and stabilized referenced to the starting structure, and local structures indicated by the torsion distribution begin to be affected by the vibration excitation. With further growth of the frequency, the effect on the local structures is increasingly significant, but the constraint to the global structure is decreased, and some irregular segments begin to be reorganized by the vibration excitation. In addition, amplitude of the vibration excitation also has influence on the polyethylene folding. Through these results, it is concluded that the vibration excitation has significant effect on the polymer molecule folding, and frequency and amplitude determine the way and the extent of the effect. With appropriate choosing of frequency and amplitude, the vibration excitation will hopefully assist the polymer folding in a more organized way. Through this research, it is possible to further study the effect of vibration excitation in the polymer crystallization even the polymer molding, and this will help us to better use the vibration technology in the polymer processing.

\section{Conflict of Interests}

The authors declare that there is no conflict of interests regarding the publication of this paper.
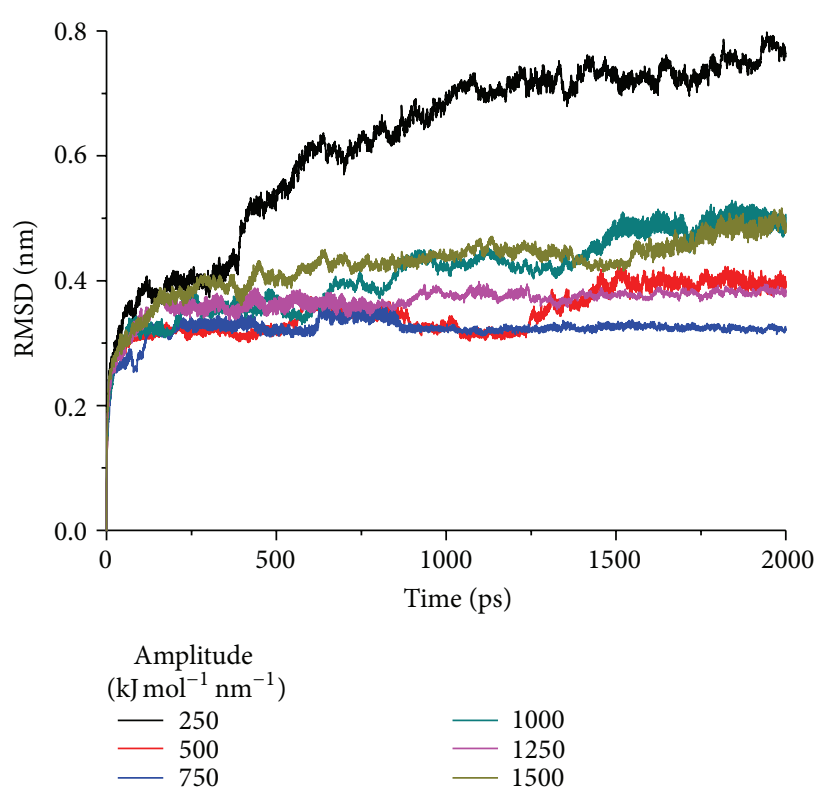

FIGURE 11: The RMSD curves of the VEMD simulations with varied amplitudes.

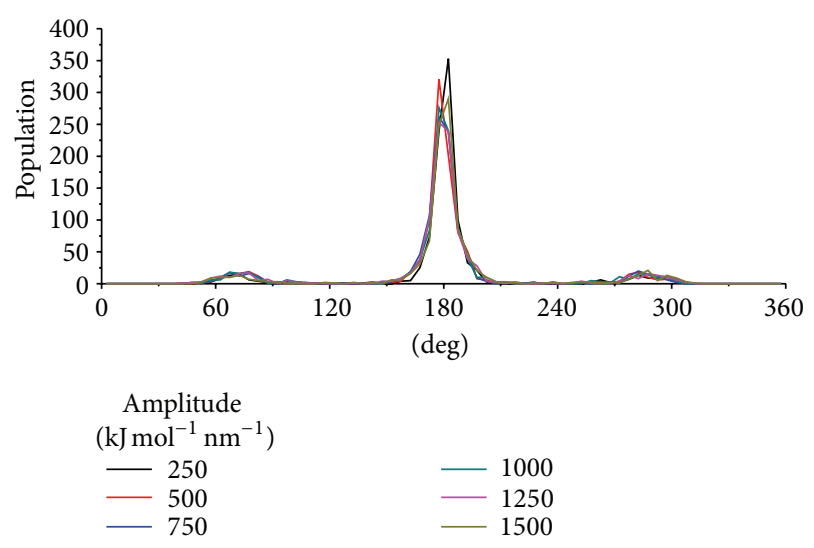

FIgURE 12: The torsion distributions of the final structures derived from the VEMD simulations with varied amplitudes.

\section{Acknowledgments}

The authors gratefully acknowledge financial support for this work from the National Basic Research Program of China (no. 2012CB025905), the National Natural Science Funds of China (no. 11202049), and the Fundamental Research Funds for the Central Universities.

\section{References}

[1] R. P. Feynman, "There's plenty of room at the bottom," Journal of Microelectromechanical Systems, vol. 1, no. 1, pp. 60-66, 1992.

[2] J. P. Ibar, "Control of polymer properties by melt vibration technology: a review," Polymer Engineering and Science, vol. 38, no. 1, pp. 1-20, 1998. 
[3] A. Kikuchi, J. P. Coulter, D. Angstadt, and A. Pinarbasi, "Localized material and processing aspects related to enhanced recycled material utilization through vibration-assisted injection molding," Journal of Manufacturing Science and Engineering, vol. 127, no. 2, pp. 402-410, 2005.

[4] A. Kikuchi, J. P. Coulter, and R. R. Gomatam, "Assessing the effect of processing variables on the mechanical response of polysytrene molded using vibration-assisted injection molding process," Journal of Applied Polymer Science, vol. 99, no. 5, pp. 2603-2613, 2006.

[5] Y. Feng, J. Qu, H. He, G. Jin, X. Cao, and J. Song, "Simulation of nonisothermal flow of melt during melting process of vibrationinduced polymer extruder," Journal of Applied Polymer Science, vol. 102, no. 6, pp. 5825-5840, 2006.

[6] Y. Li, K. Shen, and J. Zhan, "Improving rheological property of polymer melt via low frequency melt vibration," Journal of Applied Polymer Science, vol. 102, no. 6, pp. 5292-5296, 2006.

[7] B. Li, N. Zhou, and Y. Wang, "Study on properties of filling systems in plastic axial vibration injection processing," PolymerPlastics Technology and Engineering, vol. 47, no. 4, pp. 335-340, 2008.

[8] Y. Lu, H. Peng, K. Shen, and Z. Yan, “The study on the creep of calcium carbonate-filled polypropylene $\left(\mathrm{PP} / \mathrm{CaCO}_{3}\right)$ prepared at different vibration condition," Polymer Bulletin, vol. 66, no. 1, pp. 147-164, 2011.

[9] W. Tian, K. L. Yung, Y. Xu, L. Huang, J. Kong, and Y. Xie, "Enhanced nanoflow behaviors of polymer melts using dispersed nanoparticles and ultrasonic vibration," Nanoscale, vol. 3, no. 10, pp. 4094-4100, 2011.

[10] G. Kotzev, S. Djoumaliisky, M. Natova, and R. Benavente, "Vibration-assisted melt compounding of polypropylene/ carbon black composites: processability, filler dispersion and mechanical properties," Journal of Reinforced Plastics and Composites, vol. 31, no. 20, pp. 1353-1363, 2012.

[11] Y. Zhang, J. Zhang, X. Qian, P. Deng, and K. Shen, "Morphology evolution including formation of cylindrulite in isotactic polypropylene derived from periodical shear field," Polymer, vol. 53, no. 19, pp. 4318-4327, 2012.

[12] M. Imai, K. Kaji, and T. Kanaya, "Orientation fluctuations of poly(ethylene terephthalate) during the induction period of crystallization," Physical Review Letters, vol. 71, no. 25, pp. 41624165, 1993.

[13] K. Kaji, K. Nishida, T. Kanaya, G. Matsuba, T. Konishi, and M. Imai, "Spinodal crystallization of polymers: crystallization from the unstable melt," Advances in Polymer Science, vol. 191, no. 1, pp. 187-240, 2005.

[14] B. Wunderlich, "Thermodynamics and kinetics of crystallization of flexible molecules," Journal of Polymer Science B: Polymer Physics, vol. 46, no. 24, pp. 2647-2659, 2008.

[15] P. Panine, E. Di Cola, M. Sztucki, and T. Narayanan, "Early stages of polymer melt crystallization," Polymer, vol. 49, no. 3 , pp. 676-680, 2008.

[16] P. R. Sundararajan and T. A. Kavassalis, "Molecular dynamics study of polyethylene chain folding: the effects of chain length and the torsional barrier," Journal of the Chemical Society, Faraday Transactions, vol. 91, no. 16, pp. 2541-2549, 1995.

[17] C. Liu and M. Muthukumar, "Langevin dynamics simulations of early-stage polymer nucleation and crystallization," The Journal of Chemical Physics, vol. 109, no. 6, pp. 2536-2542, 1998.

[18] J. P. K. Doye, "Computer simulations of the mechanism of thickness selection in polymer crystals," Polymer, vol. 41, no. 25, pp. 8857-8867, 2000.
[19] H. Meyer and F. Müller-Plathe, "Formation of chain-bolded structures in supercooled polymer melts examined by $\mathrm{MD}$ simulations," Macromolecules, vol. 35, no. 4, pp. 1241-1252, 2002.

[20] M. Muthukumar, "Modeling polymer crystallization," Advances in Polymer Science, vol. 191, no. 1, pp. 241-274, 2005.

[21] C. Luo and J. Sommer, "Coexistence of melting and growth during heating of a semicrystalline polymer," Physical Review Letters, vol. 102, no. 14, Article ID 147801, 2009.

[22] T. Yamamoto, "Computer modeling of polymer crystallization: toward computer-assisted materials'design," Polymer, vol. 50, no. 9, pp. 1975-1985, 2009.

[23] C. Li, P. Choi, and P. R. Sundararajan, "Simulation of chain folding in polyethylene: a comparison of united atom and explicit hydrogen atom models," Polymer, vol. 51, no. 13, pp. 2803-2808, 2010.

[24] W. L. Jorgensen, D. S. Maxwell, and J. Tirado-Rives, "Development and testing of the OPLS all-atom force field on conformational energetics and properties of organic liquids," Journal of the American Chemical Society, vol. 118, no. 45, pp. 11225-11236, 1996.

[25] G. A. Kaminski, R. A. Friesner, J. Tirado-Rives, and W. L. Jorgensen, "Evaluation and reparametrization of the OPLSAA force field for proteins via comparison with accurate quantum chemical calculations on peptides," Journal of Physical Chemistry B, vol. 105, no. 28, pp. 6474-6487, 2001.

[26] B. Hess, C. Kutzner, D. van der Spoel, and E. Lindahl, "GRGMACS 4: algorithms for highly efficient, load-balanced, and scalable molecular simulation," Journal of Chemical Theory and Computation, vol. 4, no. 3, pp. 435-447, 2008. 

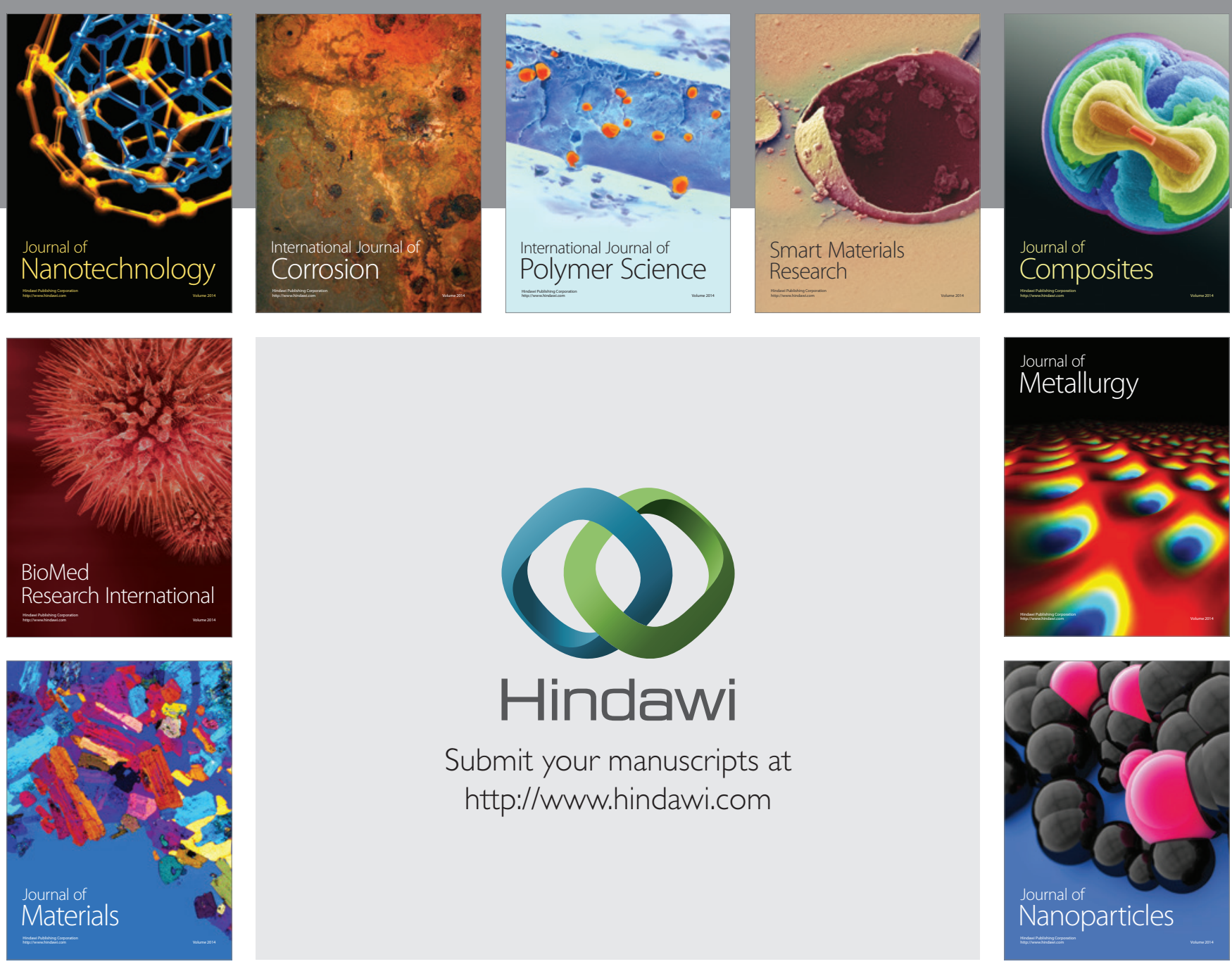

Submit your manuscripts at http://www.hindawi.com
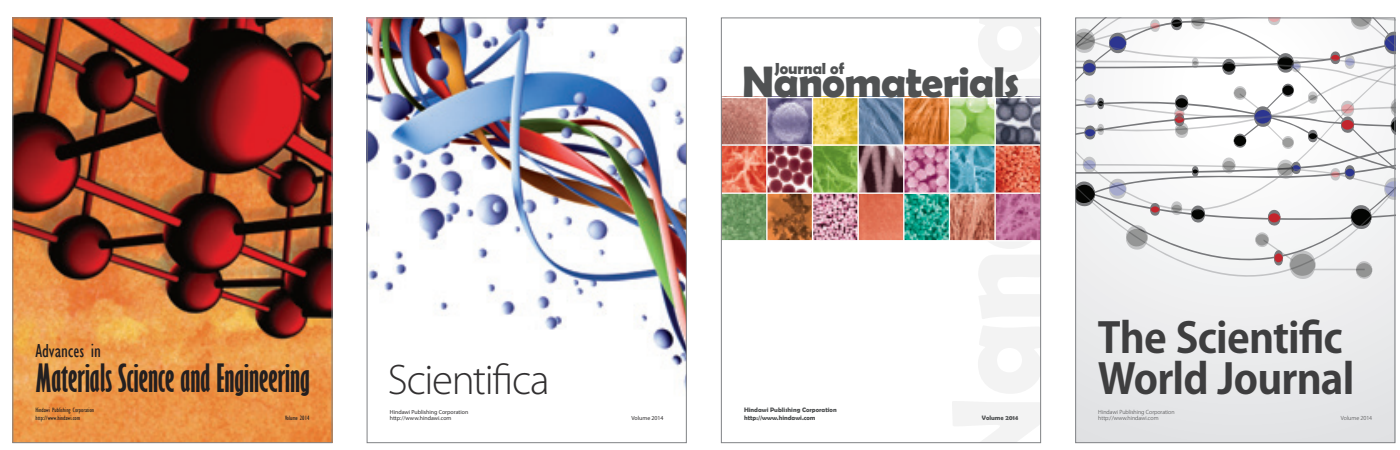

\section{The Scientific World Journal}
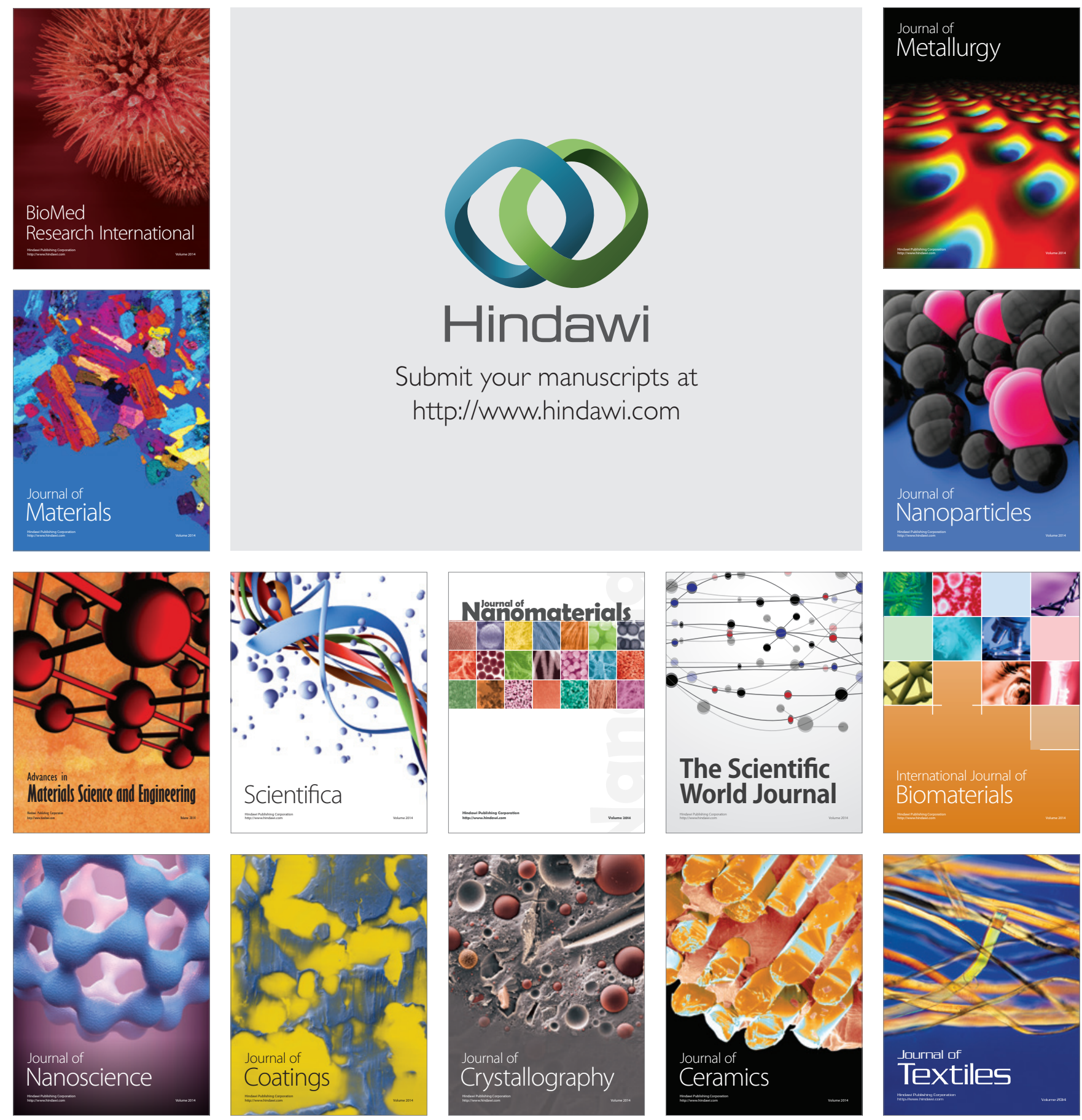